\title{
Parameter Heterogeneity Based on Virtual Capacitor Control for Suppression of the Oscillation of Radial DC Microgrid with CPLs
}

\author{
Sennian Huang ${ }^{a}$, Jingyi Liu ${ }^{b}$ \\ South China University of Technology, Guangzhou, 510640 China \\ aepsamhuangwhu@mail.scut.edu.cn, b201664492266@scut.edu.cn
}

\begin{abstract}
Keywords: radial DC micro-grid, CPL, parameter heterogeneity of filter capacitors, virtual capacitor control.
\end{abstract}

\begin{abstract}
The coupling between constant power loads (CPLs) is an important cause of system oscillation in a radial DC microgrid. First this paper deduced the equivalent circuit of a single-source dual-CPL (SSDC) system. Then, by Rouse-Hurwitz stability criterion one of the essential requirments of SSDC system stabilization is obtained, i.e. keep the parameter heterogeneity (PH) between CPLs filters is necessary. The root trajectory of the SSDC system shows that the PH of filter capacitors can improve system stability. Therefore a virtual capacitor control (VCC) of the point-of-load (POL) converter is introduced to produce the PH. The model of SSDC system was built with Matlab/Simulink. The simulation results show that the stability conditions is correct and the VCC can effectively suppress the system oscillation.
\end{abstract}

\section{Introduction}

DC microgrid has aroused considerable attention ${ }^{[1]}$, because its friendly access to distributed renewable energy and the lack of reactive power, frequency problems of traditional AC systems. Generally, $75 \% \sim 80 \%{ }^{[2]}$ DC microgrid loads access DC link through POL converters. In order to ensure normal operation of loads, load-side voltage control (LSVC) is employed. Thus, a POL converter and its load can be viewed as a CPL. Due to the negative input impedance of the CPL, its interaction with the external DC network can lead to system instability under certain conditions ${ }^{[3]}$.

For inhibition of DC microgrid instability caused by CPLs, two kinds of methods have been put forward. On the one hand, the methods based on the modification of system configuration by either changing the hardware or implementing the required component virtually in the control diagram, are presented to enhance the system stability margin ${ }^{[4,5]}$. On the other hand, the active damping methods are generally implemented by virtual components, mostly by a virtual resistor in the source-side converter control loop ${ }^{[6]}$.

Most of researches mentioned above are based on a point-to-point system. However, the practical system is often a radial DC microgrid with multiple CPLs, whose stability conditions and oscillation suppression methods should be studied necessarily. In this paper, the stability condition for stable operation of radial DC microgrid is derived by dissecting a SSDC system (multi-source can be equivalent to one source $\left.{ }^{[7]}\right)$. Then, the load-side VCC is employed to achieve the parameter heterogeneity of filter capacitors. The accuracy of stability conditions of the radial DC microgrid and the validity of VCC will be verified by Matlab/Simulink simulation.

\section{Transient Model of DC Microgrids With CPLs}

The DC microgrid shown in Fig. 1 is studied. In this system, the source-side converter is controlled by droop control and POL converters are controlled by LSVC. Each converter is controlled only with local variables. Thus a central controller and communication between converts are not necessary.

The battery is often connected to the PV output bus for suppressing the fluctuation of the PV power and the maximum utilization of renewable energy. In droop control mode, its port characteristics can be equivalent to an ideal voltage source series an impedance. Fig. 2 is the equivalent circuit of a PV 
containing an energy storage device, where $R_{\mathrm{eq}}=R_{\mathrm{v}}+R_{\mathrm{t}}$. In general, $R_{\mathrm{eq}} \gg L_{\mathrm{t}}$, then $L_{\mathrm{t}}$ can be ignored, so the equivalent circuit can be an ideal voltage source series a resistance.

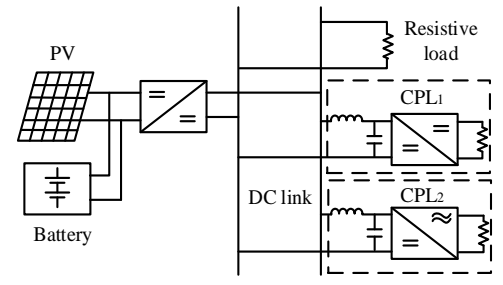

Fig. 1 Photovoltaic-battery-based DC microgrid

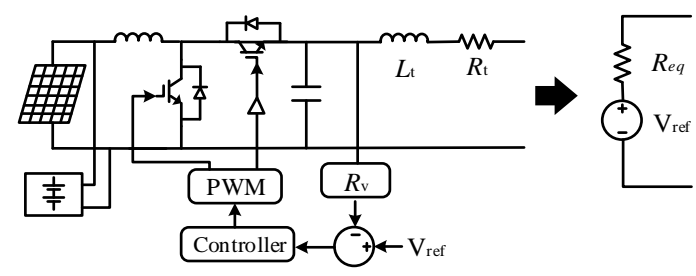

Fig. 2 Equivalent circuit of PV with energy storage

The POL converters and their loads can be regarded as CPLs (Fig. 3), because the LSVC action realize that the load-side voltage is almostly constant, thus the active power of loads is also constant. If the input voltage increases, the LSVC action reduces the input current and vice versa. The I-V relationship of the voltage controlled current source (VCCS) can be represented by
$\mathrm{i}(\mathrm{u})=\mathrm{PL} / \mathrm{u}$
$\mathrm{u}>\mathrm{Uth}$

Without loss of generality, the SSDC system in Fig. 4 is taken as an example. Its simplified equivalent circuit shown where $R$ is the resistive load, $u_{\mathrm{dc}}$ is the bus voltage, $L_{1}$ and $C_{1}$ are the inductance and capacitance of filter $1, i_{1}\left(u_{\mathrm{c} 1}\right)$ is current of the equivalent VCCS 1.
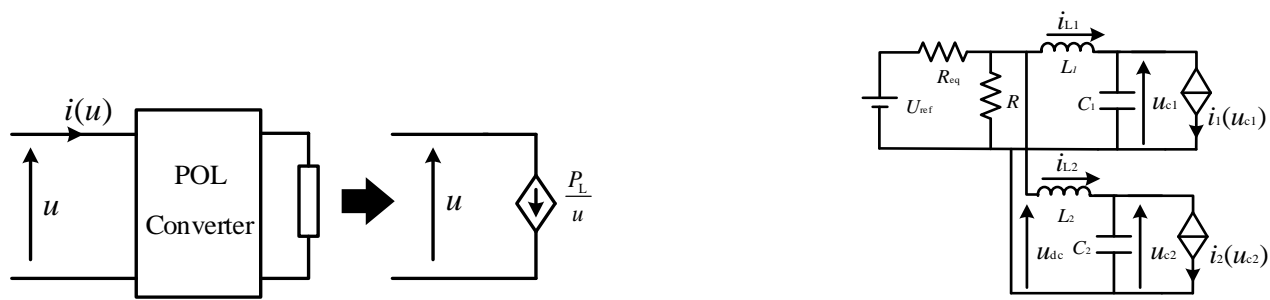

Fig. 3 Equivalent circuit of CPL

Fig. 4 Equivalent circuit of dual-CPL system

\section{Stability Analysis of Radial DC Microgrids}

The dynamic equation of this system can be derived from Fig. 4. Making it equal to zero, the equilibrium point can be solved as follow

$$
\left(i_{\mathrm{L} 1}^{*}, i_{\mathrm{L} 2}^{*}, u_{\mathrm{C} 1}^{*}, u_{\mathrm{C} 2}^{*}\right)=\left(\frac{P_{\mathrm{L}}}{u_{\mathrm{C} 1}^{*}}, \frac{P_{\mathrm{L}}}{u_{\mathrm{C} 2}^{*}}, \frac{R\left(U_{\mathrm{ref}}+\sqrt{U_{\mathrm{ref}}^{2}-4\left(P_{\mathrm{L}}+P_{\mathrm{L}}\right) R_{\mathrm{eq}}\left(R_{\mathrm{eq}}+R\right) / R}\right)}{2\left(R_{\mathrm{eq}}+R\right)}, u_{\mathrm{C} 1}^{*}\right)
$$

Solving the Jacobian matrix obtained by the dynamic equation linearization at the equilibrium point, Eigenvalues are obtained

$$
\begin{aligned}
& \lambda_{1,2}=\frac{D \pm \sqrt{D^{2}+4 B C}}{2} \\
& \lambda_{3,4}=A+\frac{D \pm \sqrt{4 A^{2}-4 A D+D^{2}+4 B C}}{2}
\end{aligned}
$$

Where $A=-R R_{\text {eq }} /\left[L_{1}\left(R+R_{\mathrm{eq}}\right)\right], B=-1 / L_{1}, C=1 / C_{1}, D=P_{\mathrm{L} 1} /\left(C_{1} u_{\mathrm{c} 1}{ }^{* 2}\right)$

Because $C_{1}, L_{1}, P_{\mathrm{L} 1}$ are always greater than zero, so $D>0$ and $B C<0$ are established. When $D+4 B C<0$, the eigenvalues $\lambda_{1,2}$ are the conjugate complex numbers with two real parts being positive. When $D+4 B C>0, \lambda_{1,2}$ are two positive real numbers. In addition, it can be seen from (3) that the eigenvalue $\lambda_{1,2}$ are independent of $\mathrm{A}$, so nothing to do with $R_{\text {eq. }}$. It means that when the parameters of two parallel CPLs are uniform, the system is always unstable no matter how $R_{\text {eq }}$ changes.

The filter capacitors are chosen as an example of the PH analysis. Suppose $U_{\text {ref }}=500 \mathrm{~V}, R_{\text {eq }}=0.2 \Omega$, $R=500 \Omega, L_{1}=L_{2}=291 \mathrm{uH}, P_{\mathrm{L} 1}=P_{\mathrm{L} 2}=50 \mathrm{~kW}$. The filter capacitances of two CPLs are $C_{1}=1000 \mathrm{uF}$, $C_{2}=1000(1+\sigma) \mathrm{uF}$, where $\sigma$ varies from -0.5 to 3 . Then eigenvalues of the system vary as Fig. 5 . When $\sigma=0$, the eigenvalues lie at four points with red triangle, which is consistent with the conclusion of (3). The two conjugate complex roots farther away from the imaginary axis are always located in the left 
half plane with the variation of $\sigma$. As for the other two roots, their real parts increases at first, then decreases and imaginary parts always decreases, which make them enter the left half plane when $\sigma \approx 1$.

In summary, the system is always unstable when the parameters of parallel CPLs are identical. The capacitance PH introduced by increasing the individual capacitance is beneficial to suppress oscillation and widen the stability margin.

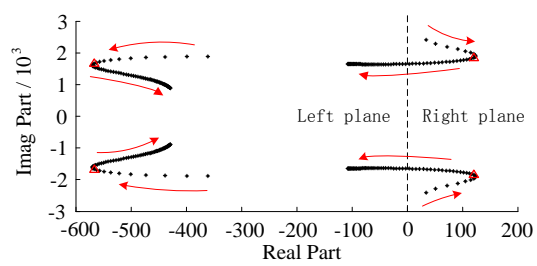

Fig. 5 Root trajectory with changing $\sigma$

\section{Virtual Capacitor Control}

The analysis above shows that the oscillation caused by consistency of filter parameters can be suppressed by the PH of the filter capacitors. However, it is high cost and not flexible to adjust capacitors in high-voltage equipments. The load-side VCC can flexibly adjust the value of $\mathrm{C}$ and improve the system stability without changing the high-voltage devices.

Fig. 6 shows a simplified system with a virtual capacitor. Assuming that there is an actual parallel capacitor $C_{\mathrm{v}}$, then the absorbed power corresponding to this capacitor is

$p(t)=u_{\mathrm{s}}(t) \cdot i(t)=u_{\mathrm{s}}(t) \cdot C_{\mathrm{v}} \frac{d u_{\mathrm{s}}(t)}{d t}$

In fact, when the virtual capacitor control is applied, $p(t)$ is absorbed by the POL converter. The VCC block diagram is shown in Fig. 7 , where $p_{\mathrm{v}}, p_{\mathrm{s}}, p_{\mathrm{s}}{ }^{*}, \omega_{\mathrm{c}}$, respectively, virtual capacitor power, CPL power, CPL power with a VCC, cutoff frequency of the low-pass filter, $T_{1}=1 / \omega_{\mathrm{c}}$. Then, according to Fig. 7, $p_{\mathrm{v}}(t)$ can be derived as

$$
P_{\mathrm{v}}(t)=C_{\mathrm{v}} L^{-1}\left(\frac{s}{1+s T_{1}} U_{\mathrm{s}}(s)\right) L^{-1}\left(\frac{U_{\mathrm{s}}(s)}{1+s T_{1}}\right)=C_{\mathrm{v}} u_{\mathrm{sff}}(t) \cdot \frac{d u_{\mathrm{sIf}}(t)}{d t}
$$

(5) is similar to (4) except that the input voltage is used to calculate the power absorbed by the virtual capacitor in (4), while the low-frequency component of $u_{\mathrm{s}}\left(u_{\mathrm{slf}}\right)$ is used to do that in (5). This improvement can effectively reduce the switching frequency and the load power fluctuation.

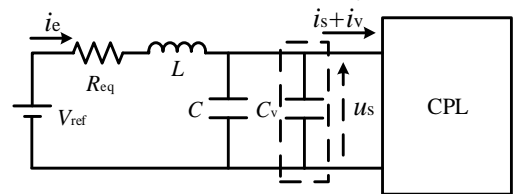

Fig. 6 The external characteristic of VCC

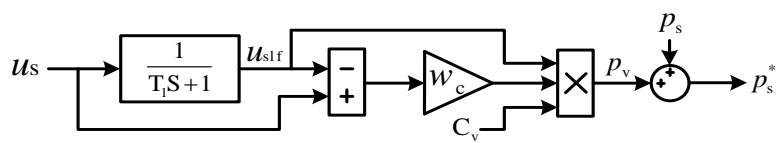

Fig. 7 Block diagram of VCC

\section{Simulation Analysis}

\subsection{Case 1}

A system shown in Fig. 4 is built in Matlab/Simulink. Two CPLs with LC filters are connected, $U_{\text {ref }}=500 \mathrm{~V}, \mathrm{R}_{\mathrm{eq}}=0.2 \Omega, R=500 \Omega, L_{1}=L_{1}=291 \mathrm{uH}, C_{1}=C_{1}=1000 \mathrm{uF}$. At $t=0.1 \mathrm{~s}$, the $\mathrm{CPL}_{1}$ of 50 $\mathrm{kW}$ is connected. At $t=0.2 \mathrm{~s}$, the $\mathrm{CPL}_{2}$ of $50 \mathrm{~kW}$ is connected. It is worth noting that two LC filters are always connected. As shown in Fig. 8, divergent oscillations of $u_{\mathrm{c} 1}, i_{\mathrm{L} 1}, u_{\mathrm{c} 2}$ and $i_{\mathrm{L} 2}$ occur after $\mathrm{CPL}_{1}$ is connected at $t=0.1 \mathrm{~s}$. The reason why the system is unstable can be found in the eigenvalues. When

$P_{\mathrm{L} 1}=50 \mathrm{~kW}, P_{\mathrm{L} 2}=\mathrm{O}$

Substituting (6) and other parameters into (3), it can be derived that $\lambda 1,2=58.6 \pm \mathrm{j} 1849$.

\subsection{Case 2}

A VCC is added to the POL converter of $\mathrm{CPL}_{2}$. According to the analysis of section 3, the virtual capacitor $C_{\mathrm{v}}$ is set to the value of $1000 \mathrm{uF}$. Considering the resonant frequency of the filter $(1854 \mathrm{rad} / \mathrm{s})$, the cutoff frequency $\omega_{\mathrm{c}}$ is set to the value of $2500 \mathrm{rad} / \mathrm{s}$. The other parameters and operations are the 
same as case 1. The voltage and current waveforms of two CPLs are shown in Fig. 9. After the CPL of $50 \mathrm{~kW}$ is connected at $t=0.1 \mathrm{~s}$ and the $\mathrm{CPL}_{2}$ of $50 \mathrm{~kW}$ is connected at $t=0.2 \mathrm{~s}$, both $u_{\mathrm{c} 1}, i_{\mathrm{L} 1}, u_{\mathrm{c} 2}$ and $i_{\mathrm{L} 2}$ can enter the steady state after oscillations, the system remains stable.

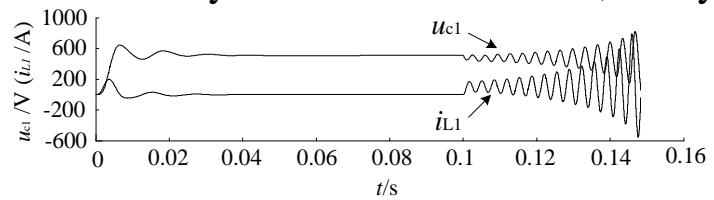

(a) CPL1

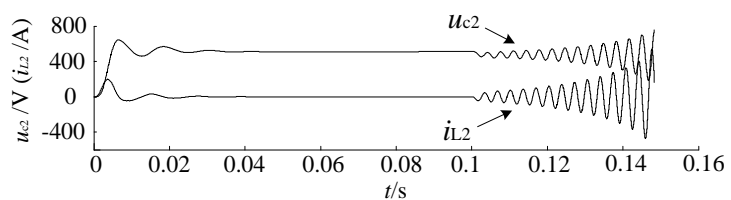

(b) CPL2

Fig. 8 Voltage and current response of CPL1 and CPL2 without VCC

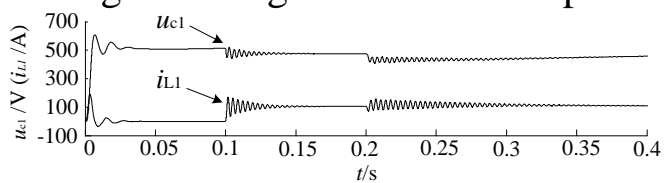

(a) CPL1

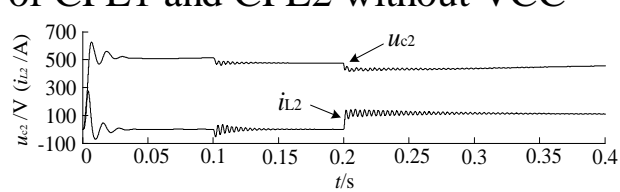

(b) CPL2

Fig. 9 Voltage and current response of CPL1 and CPL2 with VCC

\section{Summary}

In the radial DC microgrid, keeping the PH between CPL filters is a necessary condition for system stability. In case 1 this condition is certified. The comparison between case 1 and 2 shows the PH of filter capacitors based on VCC can effectively improve the stability of radial DC microgrid with multiple CPLs. Although the above derivation is done under a SSDC system, the conclusion applies to the system with multiple CPLs as well

\section{References}

[1]. Justo J J, Mwasilu F, Ju L, et al. AC-microgrids versus DC-microgrids with distributed energy resources: A review[J]. Renewable \& Sustainable Energy Reviews, Vol. 24 (2013) No. 10, p. 387-405.

[2]. RADWAN A A A, ABDEL-RADY Y I M, Linear active stabilization of converter-dominated DC microgrids[J]. IEEE Trans. Smart Grid, Vol. 3 (2012) No. 1, p. 203-216.

[3]. Wook-Jin Lee, Seung-Ki Sul, DC-link voltage stabilization for reduced dc-link capacitor inverter[C]. IEEE ECCE, San Jose, CA, USA, 2009, p. 1740-1744.

[4]. Ali Emadi, Alireza Khaligh, Claudio H. Rivetta, Geoffrey A. Williamson, Constant power loads and negative impedance instability in automotive systems: definition, modeling, stability, and control of power electronic converters and motor drives[J]. IEEE Trans. Vehicular Technology, Vol. 55 (2006) No. 4, p. 1112-1125.

[5]. Yumei Li, Xiaoming Zha, Fei Liu, Oscillation suppression of multi-source DC microgrid with multiple constant-power loads[J]. Electric Power Automation Equipment, Vol. 34 (2014) No. 3, p. 40-46.

[6]. Mingfei Wu, Dylan Dah-Chuan Lu, A Novel Stabilization Method of LC Input FilterWith Constant Power Loads Without Load Performance Compromise in DC Microgrids[J]. IEEE Trans. Power Electronics, Vol. 62 (2015) No. 7, p. 4552-4562.

[7]. André Pires Nóbrega Tahim, Daniel J. Pagano, Eduardo Lenz, Vinicius Stramosk, Modeling and Stability Analysis of Islanded DC Microgrids Under Droop Control[J].IEEE Trans. Power Electronics, Vol. 30 (2015) No. 8, p. 4597-4607. 